\title{
Efficient Deformable Motion Correction for 3-D Abdominal MRI Using Manifold Regression
}

\author{
Xin Chen ${ }^{1,2}$, Daniel R. Balfour ${ }^{1}$, Paul K. Marsden ${ }^{1}$, Andrew J. Reader ${ }^{1}$, \\ Claudia Prieto ${ }^{1}$, Andrew P. King ${ }^{1}$ \\ ${ }^{1}$ Division of Imaging Sciences and Biomedical Engineering, King's College London, U.K. \\ ${ }^{2}$ School of Computer Science, University of Nottingham, U.K.
}

\begin{abstract}
We present a novel framework for efficient retrospective respiratory motion correction of 3-D abdominal MRI using manifold regression. K-space data are continuously acquired under free breathing using the stack-of-stars radial golden-angle trajectory. The stack-of-profiles (SoP) from all temporal positions are embedded into a common manifold, in which SoPs that were acquired at similar respiratory states are close together. Next, the SoPs in the manifold are clustered into groups using the k-means algorithm. One 3-D volume is reconstructed at the central SoP position of each cluster (a.k.a. key-volumes). Motion fields are estimated using deformable image registration between each of these key-volumes and a reference end-exhale volume. Subsequently, the motion field at any other SoP position in the manifold is derived using manifold regression. The regressed motion fields for each of the SoPs are used to determine a final motioncorrected MRI volume. The method was evaluated on realistic synthetic datasets which were generated from real MRI data and also tested on an in vivo dataset. The framework enables more accurate motion correction compared to the conventional binning-based approach, with high computational efficiency.
\end{abstract}

Keywords: 3D abdominal MRI, manifold learning, manifold regression, motion correction.

\section{Introduction}

Magnetic resonance imaging (MRI) has been commonly used in different regions of interest in the body for disease detection and diagnosis. However, the acquisition speed of MRI is not sufficiently fast to permit enough data to be acquired quickly enough to reconstruct fully sampled images with high spatial resolution, especially for 3-D imaging. Therefore, image artefacts may be introduced into the reconstructed images due to motion (e.g. respiratory, cardiac, bulk motion).

In the context of this paper, only respiratory motion correction is discussed and so our focus is on the abdominal region. Breath-hold imaging is a simple and efficient solution to the problem of respiratory motion but it is not applicable to all patients and MRI sequences. Respiratory gated acquisition is a commonly used alternative [1]. With gating, acquired data is only retained when a diaphragm navigator falls within a predefined small gating window. However, gated scans normally result in a longer acquisition time due to low scan efficiency (i.e. discarded data needs to be reacquired). To increase the scan efficiency and use all (or almost all) of the acquired data for reconstruction, a number of works have either used image navigators [2] or self-gating methods [3] to group the k-space data into different motion states and applied image registration techniques to warp the data from different motion states to a reference motion 
state. A key weakness of this approach is the simplicity of the respiratory signal that typically only groups the data into a limited number of motion states (bins), which causes complex intra-cycle and inter-cycle variations in respiratory motion (known as intra-bin motion) to be averaged out. In [4] a technique was proposed for reconstructing a dynamic volume for each $\mathrm{k}$-space acquisition, but in order to use these volumes for motion correction a large number of computationally demanding image registrations would be required.

In this paper, we propose an efficient technique to retrospectively reconstruct a nonrigid motion corrected 3-D volume from $\mathrm{k}$-space data acquired in the abdominal region using a stack-of-stars (SoS) acquisition [5] under free breathing. The main contributions of this work are: (1) In contrast to state-of-the-art binning methods, instead of using a small number of motion states (usually 3 to 5), the proposed method allows the motion to be derived and corrected for each of the acquired stack-of-profile (SoP) positions, meaning that intra-bin motion can be corrected; (2) In contrast to [4], only a small number of volumes are reconstructed and registered at key SoP positions. The deformable motion fields for the remaining SoPs are estimated using manifold regression which is highly computationally efficient.

\section{Methodology}

As illustrated in Fig. 1, the proposed framework consists of k-space data acquisition, manifold embedding and clustering, motion field estimation and motion-corrected volume reconstruction, which are described in the following subsections.

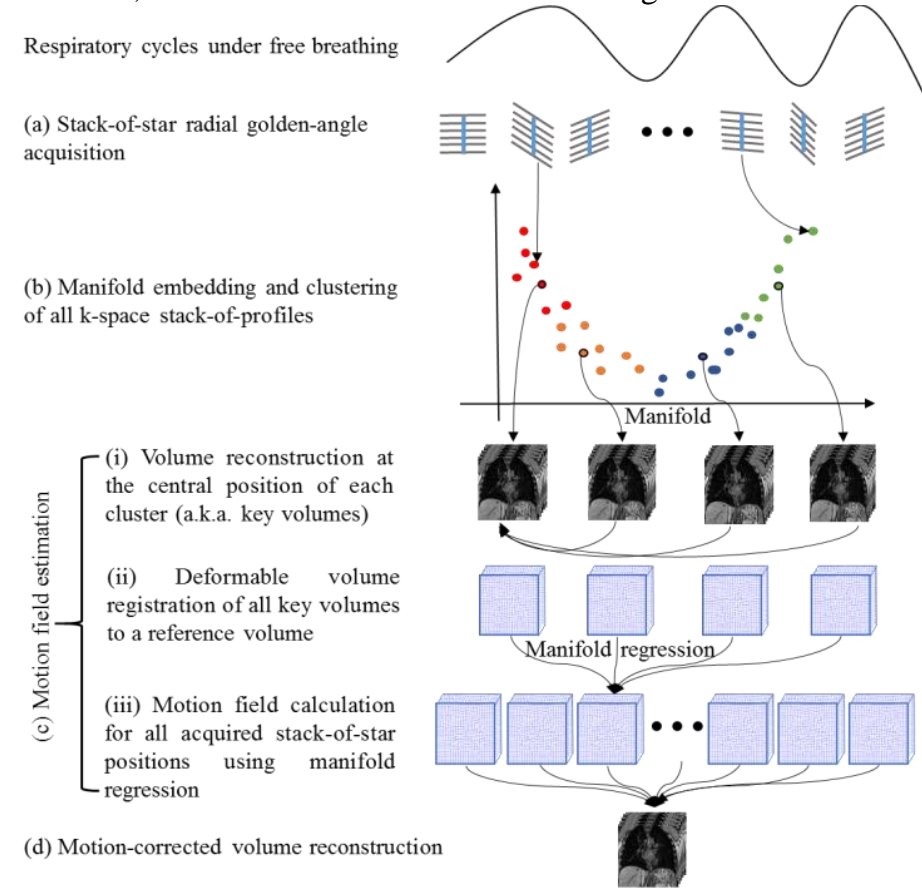

Fig. 1. Overview of the proposed framework. 


\subsection{K-space Data Acquisition}

Data acquisition is performed under free breathing using a SoS radial golden-angle (RGA) trajectory. In the SoS acquisition, one k-space profile is acquired at each of the $S$ slice positions at the same angle, followed by $S$ profiles at the next angle separated by $111.25^{\circ}$ and so on (see Fig. 1 (a)). We denote the SoP at each angle by $X_{p}($ $p \in\{1,2, \ldots, P\}, P$ is the total number of angles). Using the SoS RGA trajectory, the $\mathrm{k}$-space centre at each slice position is repetitively sampled through the entire acquisition period. The magnitudes of the central k-space lines (denoted by $x_{p}$, blue lines in Fig. 1(a)) of the SoPs correlate well with respiratory motion. We use a manifold learning method to reduce the dimensionality of $x_{p}$ from $S$ to $d(d<<S)$, and embed all the SoPs into a common manifold, where SoPs from similar motion states are close to each other. Details are described in the next subsection.

\subsection{Manifold Embedding and Clustering}

Several studies have reported that respiratory motion can be represented in a low dimensional space by applying principle component analysis (PCA) [6] or manifold learning [4] to the central k-space lines of the SoPs. Laplacian Eigenmaps [7] is used in this paper, which embeds the SoPs into a low dimensional space by preserving the local relationships in the original high dimensional space. In our work, given $P$ central $\mathrm{k}$-space lines $x_{1}, \ldots, x_{P}$ in $\mathbb{R}^{S}$, a weighted graph is constructed in which each $x_{p}$ is connected to a set of neighbouring data points $x_{q}(q \in\{1,2, \ldots, Q\}, Q$ is the number of neighbours). The weights $\left(W_{p q}\right)$ are calculated using a Gaussian kernel (with standard deviation $\sigma)$ :

$$
W_{p q}=\left\{\begin{array}{c}
e^{-\frac{\left\|x_{p}-x_{q}\right\|^{2}}{2 \sigma^{2}}}, \text { if } p, q \text { are neighbours } \\
0, \text { otherwise }
\end{array}\right.
$$

Then the coordinates $(y)$ of the low dimensional embeddings are obtained by minimising the following objective function,

$$
O(y)=\sum_{i j}\left(y_{i}-y_{j}\right)^{T}\left(y_{i}-y_{j}\right) W_{i j}=2 y^{T} L y
$$

Where $L=D-W$, and $D$ is a diagonal matrix in which the entries are column sums of $W$, calculated as $D_{i i}=\sum_{j} W_{j i}$. By adding the constraint $y^{T} D y=1$, which removes an arbitrary scaling factor in the embedding, the final $d$-dimensional vector $y$ is given by the eigenvectors that correspond to the $d$ smallest non-zero eigenvalues $(\lambda)$, in the generalised eigenvalue problem:

$$
L y=\lambda D y
$$

As illustrated in Fig. 1 (b), each point represents the location of its corresponding SoP in the manifold. Points in the manifold that are close together have similar motion states. Next, we apply the k-means algorithm to automatically group the data points according to their locations in the manifold. In practice, the k-means algorithm is run 20 times with different randomised initialisations to avoid local optima. The final clustering is the one in which the sum of distances between each of the points to their cluster centroid is the shortest. Fig. 1(b) shows the clustering results with $K=4$ for demonstration purposes. 


\subsection{Motion Field Estimation}

A key data point for each cluster is identified by calculating the mean distance of each point in the cluster to all other points in the same cluster. The point that has the shortest mean distance is used (points with black contours in Fig. 1(b)). Volume reconstruction is performed at each of these key data point locations. The reconstruction method used is the same as that described in [4], and uses $C$ SoPs that have the closest Euclidean distances in the manifold to the SoP at the key data point. The contribution of each SoP is Gaussian-weighted based on its spatial distance to the key SoP. The non-uniform fast Fourier transform [8] is applied in a slice by slice manner for volume reconstruction (Fig. 1(c-i)).

As illustrated in Fig. 1(c-ii), a reference volume is identified from the reconstructed key volumes by choosing the one which has the highest diaphragm position (i.e. the end-exhale motion state). Next, the DEEDS deformable image registration method [9] is employed to register all other key volumes to the reference volume. The motion fields for the key volumes are denoted by $m_{k}=\left\{m_{1}, \ldots, m_{K}\right\}$ ( $K$ is the number of clusters). $m_{k}$ is a vector that contains the concatenated motion displacements along the three Cartesian directions for all voxel locations at the $k^{\text {th }}$ key volume location.

Subsequently, the motion field $o_{p}$ for the $p^{\text {th }}$ (non-key position) SoP is estimated by a weighted combination of the $N$ nearest key motion fields:

$$
o_{p}=\sum_{n}^{N} V_{n_{p}} m_{n_{p}}
$$

where $n_{p}$ is the index of selected nearest key motion field for the $p^{\text {th }}$ SoP. The weight $V_{n_{p}}$ is calculated by a Gaussian kernel function (with standard deviation equal to the standard deviation of $y$ ) of the manifold distance between the $p^{\text {th }}$ SoP location and the $n^{\text {th }}$ key SoP location. In this way, motion fields for all remaining SoPs are obtained (see Fig. 1 (c-iii)) for generating the final motion-corrected volume at the reference motion state. Note that, using this approach, a motion field is estimated separately for each SoP.

\subsection{Motion-corrected Volume Reconstruction}

In [10], Batchelor et al. described a method for general motion correction in MRI. The relationship between the sampled k-space data $(s)$ and a 'motion-free' image $\left(s_{0}\right)$ can be expressed as:

$$
s=\sum_{t} A_{t} F u_{t} s_{0}=G s_{0}
$$

where $u_{t}$ is the motion in image space at acquisition time point $t . F$ represents the Fourier transform and $A_{t}$ is the $\mathrm{k}$-space sampling at time point $t$. The overall transformations can be represented by a matrix $G$. The motion corrected image $s_{0}$ is obtained by calculating the inverse of matrix $G$ and multiplying it by the acquired k-space data $s$. The authors in [10] used the conjugate gradient based algorithm to solve the matrix inversion problem. This general matrix solution is typically used when the number of motion states is small $(\sim 3-10)$ due to computational constraints. In our work, we propose to perform the motion correction based on the motion fields derived from each of the acquired SoPs, so we have hundreds of motion states, making this approach computationally infeasible. In [10], the authors also described an empirical solution that sums up the individually motion corrected images of different motion states. Although this solution is not valid when the motion direction is different to the readout direction, in our 
work the RGA acquisition is used which is less sensitive to motion due to the oversampling of the k-space center and varying readout direction. Therefore we employ the empirical solution. Specifically, each of the sampled k-space SoPs is firstly re-gridded to Cartesian coordinates with radial density compensation and by zeroing the k-space that has no acquired data. The inverse Fourier transform is then applied to the k-space data followed by image warping using the corresponding motion fields derived as described in the previous section. The final motion-corrected volume is the sum of the individually motion-corrected volumes at all the acquired SoP positions. Note that the summation is performed using the complex values.

\section{Experiments and Results}

The proposed method was evaluated on both synthetic and in-vivo datasets. The synthetic datasets were used to establish a ground truth for quantitative evaluation, and the results were compared with the binning-based motion correction (BMC) method. We also demonstrated the practical feasibility of our method using an in vivo dataset.

\subsection{Materials}

Synthetic dataset generation: Based on a respiratory gated high spatial resolution (RGHR) 3D volume and a dynamic 3D low spatial resolution sequence, the authors in [4] proposed a framework to generate high-temporal and high-spatial resolution dynamic sequences with randomised breathing cycles containing both intra- and intercycle variation. We employed this method in our experiments. To mimic the SoS acquisition, we generated 20 breathing cycles. Each breathing cycle lasted approximately $5 \mathrm{~s}$ and the interpolated volumes had a temporal resolution of $\sim 4 \mathrm{~ms}$. In total, 14000 high spatial/temporal resolution dynamic volumes containing realistic intra-cycle and inter-cycle variation were obtained with a matrix size of $250 \times 250 \times 280$ and isotropic resolution of $1 \mathrm{~mm}^{3}$. From each volume we perform a fast Fourier transform to produce k-space data. From the first volume's k-space data we extract a single profile at the first slice position and profile angle. The profiles for subsequent volumes are extracted from the next slice positions. Once all slice positions have been sampled we move on to the next profile angle and repeat the procedure. With 35 slices ( $8 \mathrm{~mm}$ slice thickness), approximately 400 SoPs were simulated. According to the Nyquist sampling theory for an image resolution of $250 \times 250$, approximately $400(250 \times \pi / 2)$ radial profiles are required to produce an aliasing free image. We generated 5 such highly realistic synthetic SoP datasets to validate our method.

In vivo dataset acquisition: A SoS acquisition with RGA trajectory was employed for data acquisition in the liver and lung region of a healthy volunteer. Data was acquired on a Philips $1.5 \mathrm{~T}$ scanner using a 28 channel-coil with T1 weighted sequence in sagittal slices. The settings were $\mathrm{TR}=4.9 \mathrm{~ms}$, TE $=2.2 \mathrm{~ms}$, flip angle $=15^{\circ}$, and acquired voxel size $2.0 \times 2.0 \times 8.0 \mathrm{~mm}^{3}$ with acquired matrix size of $176 \times 176 \times 38$. A total of 500 SoPs were acquired under free-breathing in approximately 90 seconds. 


\subsection{Results}

Synthetic dataset: The manifold embedding of a synthetic dataset is shown in Fig. 2 (a) with dimensionality of $d=2$. In our experiments the use of $d=1$ or $d>2$ did not produce better results. The colours represent the normalised head-foot diaphragm position of the ground truth. Similar coloured points grouping together indicates a good manifold embedding. The clustering result with $K=5$ is shown in Fig. 2 (b). The black circles indicate the SoP positions for key-volume reconstructions. With a fixed number of input SoPs of 400, we explored the effects of varying the number of clusters $(K)$ and the number of nearest neighbours $(N)$ for motion field regression. The reconstructed image quality was quantitatively measured based on the sum of squared differences (SSD) and peak signal-to-noise ratio (PSNR) against the ground truth. Based on one dataset, Fig. 3 (a) and (b) respectively show the SSD and PSNR values of our method using different numbers of $K$ with only the optimum number of $N$ that produced the best results, and compares with results of the BMC method. Note that the result of using $N=1$ for our method is equivalent to the BMC method, since for each of the SoPs it uses the motion field of the nearest key motion state. It can be seen from Fig. 3 that performance increases when a larger number of clusters are used, and a larger number of $N$ is required to produce a better result. The optimum number of $N$ for each cluster was found to be consistent across all five tested datasets even though their breathing patterns were significantly different. In addition, our proposed method consistently outperformed the BMC method independent of the number of clusters $(K)$. From the experiments, we conclude that the use of $K=10$ and $N=3$ achieved the best balance between reconstruction accuracy and computational time. Based on this setting, the proposed method resulted in 5.8 $\pm 2.8 \%$ smaller SSD and $0.9 \pm 0.3 \mathrm{~dB}$ larger PSNR, compared with the BMC method over the 5 synthetic datasets. The majority of the computational time was occupied by the DEEDS image registration process which was about 60 s per volume. The volume reconstruction time is about $15 \mathrm{~s}$ per volume. The time for motion correction at individual SoP locations was less than $1 \mathrm{~s}$ per SoP. Therefore, the overall computational time linearly increased with the number of clusters. As an example, the overall time for producing the final motion corrected volume for the input sequence of 400 SoPs using $K=10$ was about 20 mins on a $3.6 \mathrm{GHz}$ computer. It was about 13 mins for the same dataset using the BMC method.

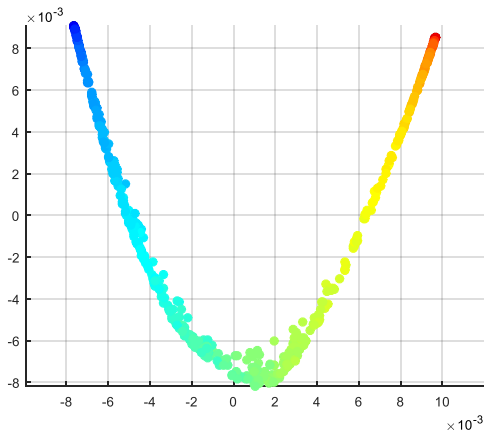

(a)

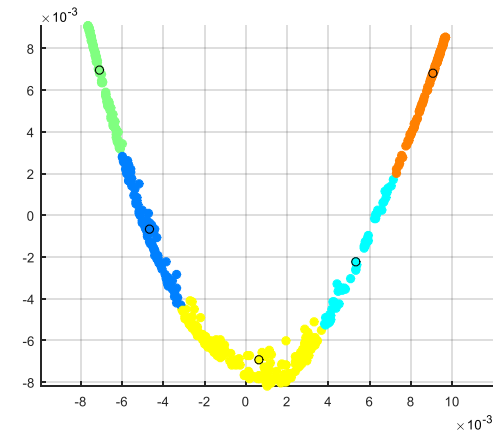

(b)

Fig. 2. (a) Manifold embeddings for a synthetic dataset (b) clustering results of $K=5$ with black circles indicating SoP positions for key volume reconstruction. 


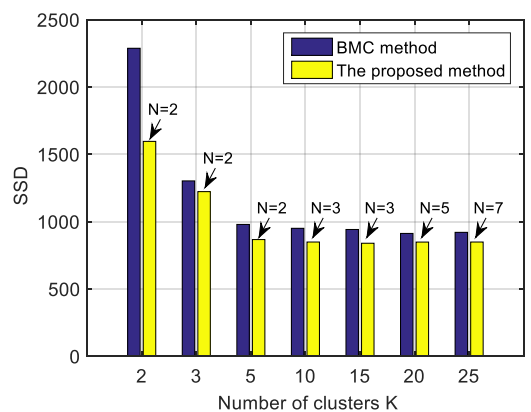

(a)

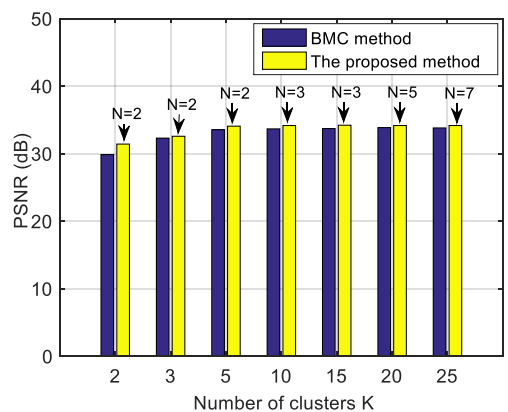

(b)

Fig. 3. (a) SSD values (b) PSNR values of the proposed method using different numbers of clusters $(K)$ with the optimum number of nearest neighbours $(N)$, compared with the binning-based motion correction method (BMC).

In vivo dataset: We applied the proposed framework to an in vivo dataset to demonstrate the practicality of the method. For the dataset described in section 3.1, the reconstructed volumes without motion correction and using our motion correction method are shown in Fig. 4 at specific slices in sagittal view and coronal view respectively. Bilinear interpolation is used for the coronal view as the slice thickness is $8 \mathrm{~mm}$. An improvement in image quality and sharpness in the motion corrected volume can be seen, particularly at those regions that are highlighted by red arrows. A sharpness measurement was used as a quantitative measure of image quality, which was measured as the average of the image gradient magnitude in the liver and liver-lung boundary (the larger the sharper). The sharpness measures for the motion corrupted volume and motion corrected volume were 0.2748 and 0.3545 respectively.

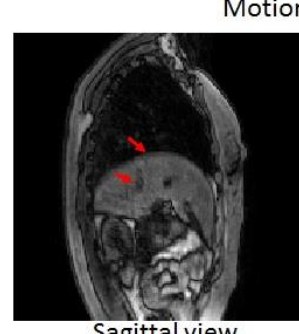

Sagittal view

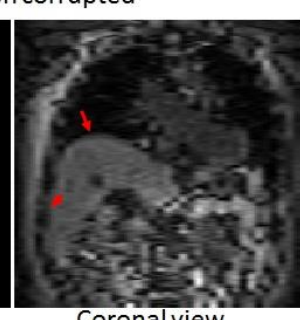

Coronalview

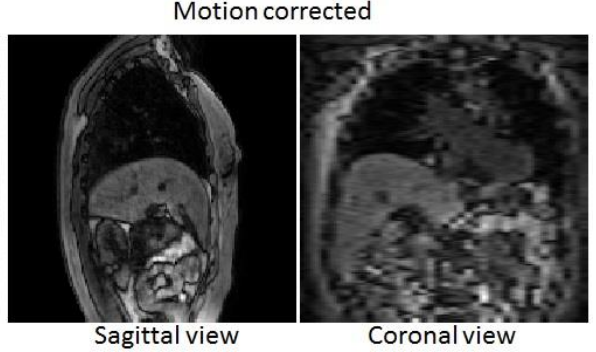

Fig. 4. Volumes reconstructed for the in vivo dataset without motion correction (left two) and with the proposed motion correction method (right two) in sagittal and coronal views.

\section{Conclusion and Discussions}

We have presented an efficient motion correction framework that is able to correct deformable motions for each acquired stack-of-profiles using a stack-of-stars acquisition under free breathing. Based on synthetic datasets, compared with the BMC method, our method achieved better performance on SSD and PSNR measures against the ground truth without compromising computational efficiency. We believe that the improved 
performance of our method is due to the fact that intra-bin motions were corrected with the individual motion fields derived using manifold regression. Our method produced a motion corrected volume for $400 \mathrm{SoPs}$, using individual motion fields for each SoP, in about 20 minutes (10 registrations). In contrast, the current state-of-the-art [4] would require 400 registrations (one for each SoP), taking approximately 7 hours. We also demonstrated the practicality of the proposed method on an in vivo dataset which achieved a good motion correction result. Our proposed method is likely to have considerable benefits over the conventional binning method when a longer MR sequence is acquired, as the intra-cycle and inter-cycle motion variations may become more significant. For example, this is the case when MRI is used for motion correction of PET in an integrated PET/MRI scenario.

\section{Acknowledgements}

This work was funded by the Engineering and Physical Sciences Research Council (Grant EP/M009319/1).

\section{References}

[1] Y. Wang, P. Rossman, R. Grimm, S. Riederer, and R. Ehman, "Navigator-echo-based realtime respiratory gating and triggering for reduction of respiration effects in threedimensional coronary MR angiography.," Radiology, vol. 198, pp. 55-60, 1996.

[2] C. Prieto, M. Doneva, M. Usman, M. Henningsson, G. Greil, T. Schaeffter, and R. Botnar, “ Highly efficient respiratory motion compensated free-breathing coronary mra using goldenstep Cartesian acquisition,” Mag. Res. Img. , vol. 41, no. 3, pp. 738-746, 2015.

[3] C. Buerger, R. Clough, A. King, T. Schaeffter, and C. Prieto, "Nonrigid motion modeling of the liver from 3-D undersampled self-gated golden-radial phase encoded MRI," IEEE Trans. on Med. Img., vol. 31, no. 3, pp. 805-815, 2012.

[4] X. Chen, M. Usman, C. Baumgartner, D. Balfour, P. Marsden, A. Reader, C. Prieto, and A. King, "High-Resolution Self-Gated Dynamic Abdominal MRI Using Manifold Alignment," IEEE Trans. on Med. Img., vol. 36, no. 4, pp. 960-971, 2017.

[5] K. Block, H. Chandarana, S. Milla, M. Bruno, T. Mulholland, G. Fatterpekar, M. Hagiwara, R. Grimm, C. Geppert, B. Kiefer, and D. Sodickson, "Towards Routine Clinical Use of Radial Stack-of-Stars 3D Gradient-Echo Sequences for Reducing Motion Sensitivity," Journal of the Korean Society of Mag. Res. in Med., vol. 18, no. 2, pp. 87-106, 2014.

[6] L. Feng, L. Axel, H. Chandarana, K. Block, D. Sodickson, and R. Otazo, "XD-GRASP: Golden-Angle Radial MRI with Reconstruction of Extra Motion-State Dimensions Using Compressed Sensing," Mag. Res. in Med., vol. 75, no. 2, pp. 775-788, 2016.

[7] M. Belkin, and P. Niyogi, "Laplacian Eigenmaps for dimensionality reduction and data representation," Journal of Neur. Comp., vol. 15, no. 6, pp. 1373-1396, 2003.

[8] L. Greengard, and J. Lee, “Accelerating the Nonuniform Fast Fourier Transform," Society for Industrial and Applied Math. Review, vol. 46, no. 3, pp. 443-454, 2006.

[9] M. Heinrich, M. Jenkinson, M. Brady, and J. Schnabel, "MRF-based Deformable Registration and Ventilation Estimation of Lung CT," IEEE Trans. on Med. Img., vol. 32, no. 7, pp. 12391248, 2013.

[10] P. Batchelor, D. Atkinson, P. Irarrazaval, D. Hill, J. Hajnal, and D. Larkman, "Matrix description of general motion correction applied to multishot images," Magn. Reson. Med., vol. 54, no. 4, pp. 1273-1280, 2005. 